\title{
The Relationship of Blood Prolactin, Cortisol and Thyroid Hormones in Prematural Newborns with Respiratory Distress Syndrome
}

\section{ISSN: 2576-9200}

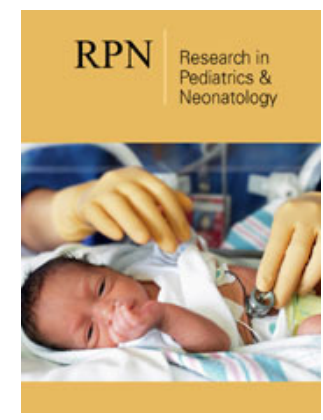

${ }^{* 1}$ Corresponding author: Baris Yilmaz, Division of Pediatric Hematology and Oncology, Turkey

Submission: 㭗 September 06, 2019

Published: 此 September 24, 2019

Volume 3 - Issue 5

How to cite this article: Emine Hekim Yilmaz, Baris Yilmaz, Deniz Bahadir Firat, Fatih Burak Kaner. Al-Sadeeq. The Relationship of Blood Prolactin, Cortisol and Thyroid Hormones in Prematural Newborns with Respiratory Distress Syndrome. Res Pediatr Neonatol. 3(5). RPN.000572.2019.

DOI: $10.31031 /$ RPN.2019.03.000572

Copyright@ Baris Yilmaz. Al-Sadeeq, This article is distributed under the terms of the Creative Commons Attribution 4.0 International License, which permits unrestricted use and redistribution provided that the original author and source are credited.

\author{
Emine Hekim Yilmaz ${ }^{1}$, Baris Yilmaz ${ }^{2 *}$, Deniz Bahadir Firat ${ }^{3}$ and Fatih Burak \\ Kaner $^{3}$ \\ ${ }^{1}$ Research and Education Hospital, Turkey \\ ${ }^{2}$ Division of Pediatric Hematology and Oncology, Turkey \\ ${ }^{3}$ School of Medicine, Turkey
}

\begin{abstract}
RDS is one of the leading causes of morbidity and mortality in preterm infants. It is well known that the main reason for RDS development is the deficiency of pulmonary surfactant. Various endogenous hormones such as prolactin, cortisol, testosterone, estradiol, estrone, thyroxine, and triiodothyronine play a major role in fetal lung maturation. Neonates with low birth weights and low gestational age are reported to have a higher risk of developing RDS than average. Several studies which investigated the relationship between thyroid hormones and RDS had shown that infants with RDS have lower free T4 (fT4) and total (tT4) serum levels than infants without RDS although both groups had similar gestational ages and birth weights. Serum prolactin level of neonates increases proportionally with their gestational ages and their birth weights.
\end{abstract}

Numerous studies have been shown infants who have lower serum prolactin levels have an increased risk of developing RDS, therefore prolactin might be an important factor in fetal lung maturation. A rise cortisol concentration in cord blood is recorded before the rise in lecithin/sphingomyelin ratio (an indicator of surfactant maturation), an inverse relationship between the cortisol concentration and RDS occurrence is found, and cortisol administration before conception in risky pregnancies seemed to speed up surfactant maturation. These findings favor the cortisol as one of the key factors in fetal surfactant maturation, therefore, making the cortisol administration a vital aspect of RDS prevention. 56 neonates without RDS and 61 neonates with RDS, in total 117 neonates younger than 37 weeks of gestational age were examined (prospectively or retrospectively). Blood samples from each of them were taken in their first 24 hours of life and TSH, fT 4, tT4, cortisol, and prolactin quantities were measured. Some statistical differences were found when they grouped according to their RDS development and birth weights. RDS found to increase mortality in infants. $5^{\text {th }}$ minute APGAR scores of RDS and non-RDS neonates compared and no statistically significant differences found. Although most infants with RDS had $5^{\text {th }}$ minute APGAR scores between 4 to 7 and most of the neonates without RDS had scores of above 7. Hospital stay for infants with RDS was significantly longer. TSH, fT4, cortisol, Tt4, and prolactin levels were compared according to the presence of RDS and tT4, cortisol, and prolactin values were significantly lower in infants with RDS. Also, TSH and prolactin levels of neonates with RDS seemed to decrease as the babies gestational age lowered. Neonates without RDS and have a gestational age of 28-30 weeks are found to have the highest cortisol level amongst other groups.

\section{Introduction}

Respiratory distress syndrome (RDS) is one of the most common diseases in premature newborns. RDS and related complications are a major cause of mortality and morbidity of premature infants [2]. RDS classically begins in the first 6 hours of life with tachypnea, dyspnea, intercostal retractions, grunting, nasal flaring, and cyanosis. It is a disease characterized by increased oxygen requirement in premature infants. Typical radiological findings accompanied by this clinical picture. There are many etiologic factors, the most important etiological factor is the gestation week. The frequency of RDS increases as gestational age decreases.

Gender, maternal diabetes, perinatal asphyxia, familial predisposition, hemolytic diseases, and hormonal changes may count among the other reasons for RDS development. This study is designed to investigate the relation of RDS occurrence in premature neonates and levels of thyroid, cortisol, and prolactin hormones measured from the blood obtained in their first 24-hours of life. 


\section{Material and Methods}

This study was performed in preterm infants who were hospitalized in Dr. Lutfi Kirdar Kartal training and research hospital's newborn intensive care unit between March 1, 2009, and September 30, 2009. We used the new Ballard Scoring System for gestational age assessment, and accordingly, prematurity was defined for infants who were under 37-weeks of gestational age.

This study was conducted on 117 patients $(n=117)$ and 65 of them (55.6\%) girls, 52 of them (44.4\%) male, boy/girl ratio was 0.8 . The blood samples were taken in the first 24 hours, from all the infants. Cortisol, prolactin, TSH, free T4, total T4 hormone levels were evaluated, then we investigated the relation of RDS and these hormones. Neonates with clinical and laboratory findings suggestive of infection, blood transfusion or exchange, major congenital anomaly, intracranial hemorrhage, pneumothorax, history of additional complications such as necrotizing enterocolitis, and maternal endocrine disorders were excluded from this study. Also, family consents were taken for the neonates in the study. Participants are divided into 2groups according to their RDS status as RDS (+) which stands for neonates with RDS and RDS (-) for neonates without RDS. RDS was diagnosed via clinical findings and presence of reticulo-granular appearance on chest X-ray. The blood sample was taken within the first 24 hours of birth in both groups and, each was placed in a dry tube and serum was separated after centrifugation at 3000 rpm for 10 minutes. All serum hormone levels were measured on the same day.

The serum tT4, fT4, TSH, prolactin, and, cortisol levels were measured via the chemiluminometric method (chemical immunoassay) in Dr. Lutfi Kirdar Kartal training and research hospital biochemistry clinic laboratory. Sandwich immunoassay method with direct chemiluminometry technology was used for measurement of serum prolactin level. Two types of antibodies were used for this study. The first is the Lite separator; includes polyclonal goat anti-prolactin antibody, and a second antibody used in its solid phase, monoclonal mice anti-prolactin antibody. The antibodies were respectively added, and prolactin measurement was done afterward. Sandwich immunoassay method with direct chemiluminometry technology was used for measurement of serum TSH level.

Two types of antibodies were used for this study. The first is the Lite separator; includes a monoclonal mouse anti-TSH antibody and a second antibody used in its solid phase, a polyclonal sheep anti-TSH antibody. These antibodies were respectively added to the serum and, hormone level measurement was made after this process. The test used to measure the serum levels of cortisol, tT4, fT4 is a competitive immunoassay method which uses direct chemiluminometric technology.

The patients' cortisol, tT4, fT4 compete with labeled hormones in the Lite reagent to connect to marked rabbit anti-cortisol, antitT4, fT4 antibodies. After antibodies were respectively added to the patient's serum, the necessary measurements were completed. Normal reference ranges of the test kit were; 58.1-140.6nmol/1 for tT4, $11.5-22.7 \mathrm{pmol} / \mathrm{l}$ for fT4, $0.35-5.50 \mathrm{mIU} / \mathrm{l}$ for TSH,
2.8-29.2 mIU/l for prolactin, 4.3-22.4 4,3 $\mu \mathrm{g} / \mathrm{dl}$ for cortisol was. The data obtained from the research transferred to the electronic platform and, it was analyzed using SPSS 16.0 (Statistical Package for the Social Sciences). The data were tested for compliance with normal distributions and, it was found to be appropriate, therefore, parametric tests were used as the significance test. In the analysis and evaluations for frequency distributions, prevalence criteria used chi-square, for independent groups t-test and one-way analysis of variance was used. (?) The level of statistical significance was accepted at $\mathrm{p}<0.05$.

\section{Results}

In total 117 neonates were evaluated and RDS was detected in $56(47.9 \%)$ of cases, and RDS was not detected in $61(52.1 \%)$ patients. Of the 56 patients with RDS, 28 (50\%) were female and 28 $(50 \%)$ were male. Of 61 patients without RDS, 37 (60.6\%) patients were female and 24 (39.4\%) were male (Table 1). According to birth weights, 17 (14.5\%) neonates were below 1000gr, 34 (29.1\%) were between 1000 and 1500gr, and 66 (56.4\%) weight over $1500 \mathrm{gr}$ (Table 1). When the babies were grouped according to their gestational age, 6 (5.1\%) subjects were below the 28 weeks of gestational age, $15(12.8 \%)$ subjects were between the 28-30 weeks of the gestation age, 57 (48.7\%) subjects were between the 34-37 gestation weeks. 25 neonates $(21.4 \%)$ had born with normal vaginal delivery and, 92 (78.6\%) had born via cesarean section (Table 1). According to their $5^{\text {th }}$ minute APGAR score, $3(2,65)$ of them had below 4 points, $57(48,7 \%)$ subjects had between 5-7 point and, $57(48,7)$ subjects had more than 7 points. All subjects evaluated with respect to their gestational week and birth weight, $92(78,6 \%)$ subjects were described as preterm appropriate for gestational age (AGA), and $25(21,4 \%)$ subjects were described as preterm small for gestational age SGA 28 (\%23.9) patients of all had decreased (Table 1).

Table 1: Demographic characteristics of newborns, who are including our study.

\begin{tabular}{|c|c|c|c|}
\hline Characteristics & Categories & $\mathbf{n}$ & $\%$ \\
\hline \multirow{3}{*}{ Gender } & Girl & 65 & 55,6 \\
\hline & Boy & 52 & 44,4 \\
\hline & $<1000 \mathrm{~g}$ & 17 & 14,5 \\
\hline \multirow{4}{*}{ Weight } & $1000-1500 \mathrm{~g}$ & 34 & 29,1 \\
\hline & $>1500 \mathrm{~g}$ & 66 & 56,4 \\
\hline & $<28 w$ & 6 & 5,1 \\
\hline & $28-30 w$ & 15 & 12,8 \\
\hline \multirow{2}{*}{ Ballard Score } & $31-33 w$ & 39 & 33,3 \\
\hline & $34-37 w$ & 57 & 48,7 \\
\hline \multirow{3}{*}{ Mode of delivery } & Vaginal delivery & 25 & 21,4 \\
\hline & Cesarian section delivery & 92 & 78,6 \\
\hline & $<4$ & 3 & 2,6 \\
\hline \multirow{2}{*}{ APGAR 5 min score } & 04-Jul & 57 & 48,7 \\
\hline & $>7$ & 57 & 48,7 \\
\hline
\end{tabular}




\begin{tabular}{|c|c|c|c|}
\hline \multirow{2}{*}{ RDS } & Present & 56 & 47,9 \\
\cline { 2 - 4 } & None & 61 & 52,1 \\
\hline \multirow{2}{*}{ Preterm } & AGA & 92 & 78,6 \\
\cline { 2 - 4 } & SGA & 25 & 21,4 \\
\hline \multirow{2}{*}{ Exitus } & Yes & 28 & 23,9 \\
\cline { 2 - 4 } & No & 89 & 76,1 \\
\hline Total & & 117 & 100,0 \\
\hline
\end{tabular}

The mean values of measured fT4, tT4, TSH, cortisol, and prolactin are demonstrated in Table 2. When the RDS (+) babies and RDS (-) babies were compared in the aspect of birth weights, there was a significant statistical difference $(\mathrm{p}<0.0001)$ between them. The birth weights of RDS (-) were found to be significantly higher than RDS (+) subjects. In terms of mortality rate results demonstrate a significantly higher rate $(\mathrm{p}<0.0001)$ in RDS $(+)$ group. The mortality rate was $9,8 \%(\mathrm{n}=6)$ in RDS $(-)$ group and, $39,3 \%(n=22)$ was in RDS (+) group. When the distribution of babies according to gestational age and birth weight were analyzed in terms of RDS presence, no statistically significant difference was found. ( $p>0.05)$. When the $5^{\text {th }}$ minute APGAR scores of RDS $(+)$ and RDS (-) subjects were compared, in 44 subjects $(72,1 \%)$ of
61 RDS (-) neonates scored higher than 7 points and, 41 subjects $(73,2 \%)$ of 56 RDS (+) neonates scored between to 4-7 points. When the gestational age of the babies with and without RDS was compared, there was a significant statistical difference between the two groups $(\mathrm{p}<0.0001)$. The gestation weeks of babies, who are with RDS were significantly lower (Table 3). When the duration of hospitalization of RDS and non-RDS babies were compared, a high significantly significant difference was found ( $p<0.0001)$. The duration of hospitalization of RDS was significantly longer (RDS: $22,95 \pm 19,81$ days and non-RDS: $11,9 \pm 9,9$ days). Duration of hospitalization varies between 1 and 81 days. When TSH, tT4, fT4, cortisol, and prolactin levels were compared, tT4, cortisol, and prolactin levels were significantly different $(\mathrm{p}<0,05)$ and, tT4, cortisol, and prolactin were significantly lower in infants with RDS (Table 4). We have shown that in the preterm infants with RDS, Serum TSH and prolactin levels were significantly decreased with earlier gestational age $(\mathrm{p}<0,05)$. We saw plasma cortisol level increased until the $34^{\text {th }}$ gestational week, but in the later weeks of gestational age, this increase was not detected. No statistically significant difference was found between weeks when cortisol levels were compared.

Table 2: Hormone levels of newborns.

\begin{tabular}{|c|c|c|c|c|c|}
\hline & Free T4 & TSH & Total T4 & Cortisol & Prolactin \\
\hline Mean & 16,36 & 17,54 & 106,37 & 13,40 & 242,46 \\
\hline Median & 15,60 & 12,20 & 103,20 & 31,58 & 225,60 \\
\hline Std Error & 6,78 & 17,89 & 38,27 & 0,79 & $9,49,53$ \\
\hline Minimum Level & 1,90 & 0,70 & 29,00 & 216,50 & 496,00 \\
\hline Maximum Level & 58,60 & $>100,00$ & 187,10 & & 9 \\
\hline
\end{tabular}

Table 3: Birthweights of newborns.

\begin{tabular}{|c|c|c|c|c|}
\hline \multirow{2}{*}{ Weight } & \multicolumn{3}{|c|}{ RDS } \\
\cline { 2 - 5 } & \multicolumn{2}{|c|}{ Present } & \multicolumn{3}{c|}{ None } & n \\
\hline & n & \% & 1 & 1,6 \\
\hline $1000 \mathrm{~g}$ & 16 & 28,6 & 10 & 16,4 \\
\hline$>1500 \mathrm{~g}$ & 24 & 28,6 & 50 & 82,0 \\
\hline Total & 16 & 100,0 & 61 & 100,0 \\
\hline
\end{tabular}

Table 4: Hormone levels of newborns, with and without Rds.

\begin{tabular}{|c|c|c|c|c|c|c|}
\hline Hormone & RDS & $\mathbf{n}$ & Mean & SE & $\mathbf{t}$ & $\mathbf{p}$ \\
\hline \multirow{2}{*}{ TSH } & Present & 56 & 19,47 & 22,83 & \multirow{2}{*}{1,091} & \multirow{2}{*}{0,278} \\
\hline & None & 61 & 15,77 & 11,59 & & \\
\hline \multirow{2}{*}{ Total T4 } & Present & 56 & 93,23 & 34,62 & \multirow{2}{*}{3,756} & \multirow{2}{*}{$<0,0001$} \\
\hline & None & 61 & 118,44 & 37,72 & & \\
\hline \multirow{2}{*}{ Free T4 } & Present & 56 & 15,80 & 7,51 & \multirow{2}{*}{0,852} & \multirow{2}{*}{0,396} \\
\hline & None & 61 & 16,87 & 6,06 & & \\
\hline
\end{tabular}




\begin{tabular}{|c|c|c|c|c|c|c|}
\hline \multirow{2}{*}{ Cortisol } & Present & 56 & 11,68 & 9,87 & \multirow{2}{*}{4,298} \\
\cline { 2 - 6 } & None & 61 & 35,10 & 39,15 & $<0,0001$ \\
\hline \multirow{2}{*}{ Prolactin } & Present & 56 & 207,84 & 151,00 & 2,450 \\
\cline { 2 - 6 } & None & 61 & 274,24 & 142,05 & 0,016 \\
\hline
\end{tabular}

\section{Discussion}

RDS is a syndrome due to surfactant deficiency in preterm infants, it is still an important problem in terms of perinatal mortality and morbidity despite improvements in treatment and prevention [2,3]. It is known that RDS is more common in neonates with low birth weight and gestational age. RDS has more commonly seen in premature infants, who are low gestational age and low birth weight at some studies, which are Rainer et al. [4] and Fujiwara et al. [5]. We have seen in our study, RDS was significantly higher in newborns with low birth weight and gestational age. Various hormones such as thyroxine, triiodothyronine, prolactin, cortisol, estradiol, testosterone is known to play a role in fetal lung maturation.

In some studies of thyroid function in the postnatal period of preterm infants with RDS shown, these infants, who are with RDS cases decreased T3, T4, and fT4 levels compared to healthy preterm infants [5,6]. It has been reported that postnatal T3 and T4 increase in RDS infants did not occur or even decreased in the first 24 hours and T3 and T4gradually increased in the following weeks. In addition, low levels of postnatal TSH increase in RDS infants and normal or low TSH response to TRH suggest that maturation at hypothalamic and/or pituitary levels are not enough [4]. Redding et al. [8] demonstrated that premature newborns, who developed RDS in their follow-up had low T4 value in cord blood. On the other hand, a later study had been said, there was no significant difference of T3, T4, TSH and TBG concentrations in cord blood of term and preterm babies [7]. Stahnke et al. [9] was said that depressed thyroid hormone levels could be the result of RDS. Some previous studies have discussed whether hypothyroidism after birth is the result of hypothyroidism, low values observed at birth or the result of RDS due to hypothyroxinemia in babies with RDS. However, recent studies have shown that thyroid hormone increases are normal at birth in infants with RDS and at the postnatal first week there is a decrease in thyroid hormone levels compared to healthy preterm infants and the respiratory distress caused by lack of surfactant is thought to impair thyroid functions $[6,10,11]$.

A double-blind study by Chowdhry et al. [12] had shown no beneficial effect for RDS development of T4 treatment on very low birthweight pre maturities. [12] Chopra et al. [13] alleged that the decrease in serum T3 level and increase in the rT3 level were aimed at slowing the metabolism during the disease to protect the body. A study had shown, which is performed by Tanaka et al. found that the incidence of RDS decreased as the gestational age increased, and it had shown serum fT4 and TSH levels decreased as the gestational age decreased [14]. In addition, the study showed as the birth weight and gestation age increased, the TSH level increased, and as birth weight and APGAR score increased, the fT4 level increased.
And the same study had shown the incidence of RDS is closely related to gestational age, type of delivery, APGAR score and birth weight, but irrelevant with sex and level of plasma fT4.

Although it was not statistically significant, there was a slight decrease in TSH levels in patients with RDS. In addition, fT4 levels were found to be low in infants with asphyxia. As a result, the study had not shown the relationship between RDS and the level of plasma TSH and fT4. In our study, we evaluated the serum TSH, fT4, tT4, cortisol and prolactin levels of 117 infants with and without RDS. We found that $47.7 \%$ of babies have developed RDS. In previous studies, it was emphasized that gender was a factor affecting the appearance of RDS, 1.5-2 times more frequently in boys than in girls, but in our study, the female/male ratio was found nearly equal in RDS cases. Accordingly, in our study, gender was not effective in RDS development. We found that the incidence of RDS increased as the birth weight and gestational week decreased. These findings were consistent with the literature $[1,15]$.

Perinatal asphyxia is known to increase the incidence of RDS [16-18]. In the APGAR assessment, a value of 8-10 indicates that the child is in good condition, children between 4-7 should be followed closely, while values below four indicate that the condition of the baby is serious. Our study has not shown a statistically significant difference in the 5th minute APGAR score between RDS and nonRDS patients. We found no significant difference between TSH and fT4 levels in babies with and without RDS, whereas tT4 levels were significantly lower in babies with RDS. When our cases were grouped according to gestation weeks; we found serum tT4 level has not been significantly different between 28 weeks to 28-30, 31-33, and 34-37 weeks. While the TSH level did not change significantly between babies with and without RDS, TSH level decreased as the gestation week decreased. We showed that the TSH level was significantly lower in patients with RDS, at 28 weeks than 28-30 weeks, at 28-30 weeks than 31-33 weeks and, at 31-33 weeks than 34-37 weeks. Parker et al. [19], Andgrosso et al. [20] studied that babies who developed RDS, serum prolactin levels were reported to be lower compared to babies with normal lung function at the same gestational week. The serum PRL level is increased in proportion to gestation week and birth weight. In a study conducted by Parker CR et al. 543 newborns' cord blood, PRL levels were measured, it was concluded that the risk of RDS was higher in patients with low PRL, and PRL could be effective on fetal lung development.

Grosso DS et al. [20] demonstrated significantly lower plasma PRLlevels in a group of infants with RDS compared to infants without RDS on a study of total 782 newborns (gestational age 30-33 week) [7-31]. There was no significant difference between the groups who developed and did not develop RDS in the gestational week 34-36. Turhan et al. [27] showed in a study with 113 newborns including 
who was born in 24-42 weeks of gestational age, infants' umbilical cord cortisol and prolactin levels measured and, they found infants who were born in 24-37week gestational age prolactin levels were significantly lower in RDS developed infants than non-RDS infants. In another study by Turhan et al. [27] 113 neonates who were born at 24-42 weeks of gestation, measured serum prolactin and cortisol levels in infants umbilical cord blood and, serum prolactin levels were found to be significantly lower in newborns who developed RDS between 24 and 37 weeks of age than to those without RDS [5].

In a study by Dane et al. []on 170 pregnant women, 42 of all babies $(24.7 \%)$ were determined to develop RDS. Prolactin levels were measured in cord blood. When the relationship between gestational week and umbilical cord prolactin values were examined, prolactin values were found to be statistically significant in comparison to weeks [21]. Prolactin levels of babies with RDS were found to be below the mean prolactin level for gestational weeks. Smith et al. studied on 57 premature newborns, who were born gestational weeks ranging from 26-36 week, RDS was developed in 23 (40\%) babies who were included in this study.

The mean prolactin level was found to be $140 \mathrm{ng} / \mathrm{ml}$ in RDS patients and $276.4 \mathrm{ng} / \mathrm{ml}$ in non-RDS patients. It has been propounded that prolactin may have to play a role in fetal lung development. Schober et al. [31] measured cord blood prolactin on 30-41week-old 29 healthy babies and 28-31week-old 7 RDS developed babies. PRL levels were increased in accordance with the age of gestation, it was concluded that there was no effect on the development of RDS [22].

In our study of 117 newborns who were born under thirtyseven completed gestational weeks, in the first 24 hours blood tT4, TSH, fT4, prolactin, and cortisol levels, we found that prolactin levels were significantly different between RDS and non-RDS group. In the RDS group, PRL values were found to be significantly lower than that of 28-30 weeks, 28-30 weeks, 31-33 weeks, and 31-33 weeks, respectively. In the non-RDS group, PRL values at 2830 weeks were lower than the values of 31-33 weeks, whereas the values at 31-33 weeks were higher than the values at 34-36 weeks. These differences were not statistically significant.

An increase in the concentration of cortisol before the increase in the ratio of lecithin/sphingomyelin, which shows that the surfactant matures in amniotic fluid. Numerous studies have shown that blood cortisol levels are low in premature infants, especially those who develop RDS. It has been supported by a large number of studies that pre-treatment corticosteroids given to the preterm infant were effective in preventing RDS and it reduced neonatal mortality [23-26]. In a study, Turhan et al. [27] measured 113 newborns' cord cortisol in 24-42 weeks of gestation. The possible role of fetal cortisol on surfactant lipid synthesis in fetal lung tissue and the risk of developing RDS were higher in newborns with low serum cortisol levels. In the same study, cord cortisol showed a significant correlation with gestational age and birth weight. Murphy et al. [28] showed that cortisol levels in cord blood were low in infants who developed RDS. Hercz et al. [29], gathered samples of maternal serum and umbilical cord blood, looked at cortisol levels and found that cortisol levels in both maternal and cord blood were lower in newborns who developed RDS.

Gürses et al. [30] worked in a study with 20 RDS and 20 control groups, no statistically significant difference was found between the cord blood cortisol levels of the babies with and without RDS. In the same study, a significant difference in weight was found between RDS infants and control group. In our study, the statistically significant difference in weight was found between RDS and nonRDS babies. When the gestational age of the RDS group and control group were compared in the same study, no statistically significant difference was found between the two groups. In the same study, $40 \%$ of RDS infants died and, in our study, 39.3\% of RDS infants died. In our study, blood samples gathered from 56 patients with RDS, 61 patients with non-RDS in the first 24 hours. The cortisol levels in the blood samples were significantly lower in babies with RDS; When the infants were grouped according to gestation weeks, no significant difference was found between the gestational week and cortisol level. When the relationship between the gestational week and blood cortisol levels of non-RDS babies were examined, at gestational week 28-30, blood cortisol levels were found to be significantly higher than the other groups. There were no cases less than 28 weeks in the non-RDS group.

In conclusion, tT4, cortisol, and prolactin levels were significantly lower in infants with respiratory distress syndrome compared to the non-RDS group. There was no significant difference in TSH between RDS and non-RDS infants. When non-RDS babies were grouped according to gestational weeks, the cortisol level was significantly higher in 28-30 weeks compared to other weeks. We think that the preterm RDS infants should be evaluated for thyroid functions due to thyroid function changes similar to the euthyroid patient syndrome seen in term babies. Considering that the transition of hormones between fetal, maternal and amniotic compartments is limited, we think that intrauterine fetal therapy with prolactin, cortisol, estrogens, triiodothyronine, hormones such as triiodothyronine, or pharmacological agents that increase the serum levels of these hormones is a subject open to research.

\section{Contributors}

EHY: data collection, analysis of all data and writing this manuscript, BY: analysis of all data and revision of the manuscript, DBF and FBK: rewived the manuscript and translation to English.

\section{References}

1. Dağoğlu T, Ovali F (2007) Neonatology. In: (2nd edn), Nobel Medical Bookstores, India.

2. Farrell PM, Avery ME (1975) Hyaline membrane disease. Am Rev Respir Dis 111(5): 657-688.

3. Avery ME, Mead J (1959) Surface properties in relation to atelectasis and hyaline membrane disease. AMA J Dis Child 97(5): 517-523.

4. Dhanireddy R, Smith YF, Hamosh M, Mullon DK, Scanlon JW, et al. (1983) Respiratory distress syndrome in the newborn: Relationship to serum prolactin, thyroxine, and sex. Biol Neonate 43: 9-15. 
5. Mercado M, Yu VYH, Francis I, Szymonowicz W, Gold H, et al. (1988) Thyroid function in very preterm infants. Early Human Dev 16: 131-141.

6. Wilson DM, Hopper AO, McDougall IR, Bayer MF, Hintz RL, et al. (1982) Serum free thyroxine values in the term, premature and sick infants. J Pediatr 101(1): 113-117.

7. Redding RA, Pereira C (1974) Thyroid function in respiratory distress syndrome (RDS) of the newborn. Pediatrics 54(4): 423-428.

8. Klein RZ, Carlton EL, Faix JD, Frank JE, Hermos RJ, et al. (1997) Thyroid function in very low birth weight infants. Clin Endocrinol 47(4): 411417.

9. Stahnke N, Stenzel E, Hellwege H (1986) Thyroid function in premature with the respiratory distress syndrome. Acta Endocrinol Suppl (Copenh) 279: 354-360.

10. Hadeed AJ, Asay LD, Klein AH (1981) Significance of transient postnatal hypothyroxinemia in premature infants with and without respiratory distress syndrome. Pediatrics 68(4): 494-498.

11. Cuestas RA, Lindall A, Engel RR (1976) Low thyroid hormones and respiratory distress syndrome in the newborn. Studies on cord blood. New Engl J Med 295(6): 297-302.

12. Chowdhry P, Scanlon JW, Auerbach R, Abbasi V (1984) Results of a controlled double-blind study of thyroid replacement $n$ very low-birthweight premature infants with hypothyroxinemia. Pediatrics 73(3): 301-305.

13. Tanaka K, Shimizu T, Hosaka A, Tokita A, Shiga S, et al. (2007) Serum free T4 and thyroid stimulating hormone levels in preterm infants and the relationship between these levels and respiratory distress syndrome. Pediatr Int 49(4): 447-451.

14. Stoll BJ, Dudell GG (2007) Respiratory distress syndrome. In: Behrman R, Kleigman R, Jenson H (Eds), (18 ${ }^{\text {th }}$ edn) Nelson Textbook of Pediatrics, India, pp. 731-741.

15. Schaffer AI (1984) Disease of the newborn, pp. 133-137.

16. Tuncer M (1983) Hyalen membrane disease. Journal of Additives Hacettepe 4: 1000 .

17. Vaughan VC, Berhman ER (1983) Textbook of pediatrics Philadelphia, USA, pp. 322-416.

18. Balamkar R, Shrikhande D (2015) Surfactant replacement therapy in neonatal respiratory distress syndrome: case control study in rural hospital, Loni, India. Journal of Pharmaceutical, Biological and Chemical Sciences 6(5): 1123-1128.
19. Parker CR, Macdonald PC, Guzick DS, Porter JC, Rosenfeld CR, et al. (1989) Prolactin levels in umbilical cord blood of human infants: Relation to gestational age, maternal complications, and neonatal lung function. Am J Obstet Gynecol 161(3): 795-802.

20. Grosso DS, Macdonald CP, Thomasson JE, Christian CD (1980) Relationship of newborn serum prolactin levels to the respiratory distress syndrome and maternal hypertension. Am J Obstet Gynecol 137(5): 569-574.

21. Dane C, Beșe T, Demirkıran F, Worker H, Aksu F, et al. (1996) The effect of umbilical cord prolactin level on fetal lung maturation in pregnancies without maternal pathology. T Klin Gynecol Obst 6(3): 216-221.

22. Schober E, Simbruner G, Salzer H, Husslein P, Spona J, et al. (1982) The relationship of prolactin in cord blood, gestational age and respiratory compliance after birth in newborn infants. J Perinat Med 10(1): 23-26.

23. Crowley P (2000) Prophylactic corticosteroids for preterm birth. Cochrane Database Syst Rev, p. CD000065.

24. Brosnan PG (2001) The hypothalamic-pituitary axis in the fetus and newborn. Semin Perinatol 25(6): 371-384.

25. Kinalski M, Sledzievski A, Kretowski A (2000) Intrauterine stimulation for fetal respiratory system maturation; Benefits and risks. Wiad Leak 53: 538-545.

26. Ballard PL (1980) Hormonal influences during fetal lung development. Ciba Found Symp 78: 251-274.

27. Turhan N, Consultant N, Beysanoglu M, Dogu N, Gokmen O, et al. (1993) Umbilical cord prolactin and cortisol levels: correlation with RDS and gestational week. Anatolian J Gynecol Obst 3: 203-206.

28. Murphy BEP (1974) Cortisol and cortisone levels in the cord blood at the delivery of infants with and without the RDS. Am J Obstet Gynecol 119(8): 1112-1120.

29. Hercz P, Várady E, Ungár L, Siklós P, Büky B, et al. (1987) Respiratory distress syndrome: Steroid and peptide hormone levels in maternal venous blood an umbilical vein and artery. Acta Pediatr Hung 28(1): 1721.

30. Gurses N, Sarioglu K, Baysal K (1991) Cord blood insulin, cortisol and glucose levels in premature infants with RDS. Turk J Resc Med Sci 9: 356361 .

31. Smith YF, Mullon DK, Hamosh M, Scanlon JW, Hamosh P, et al. (1980) Serum prolactin and respiratory distress syndrome in the newborn. Pediatr Res 14(2): 93-95. 